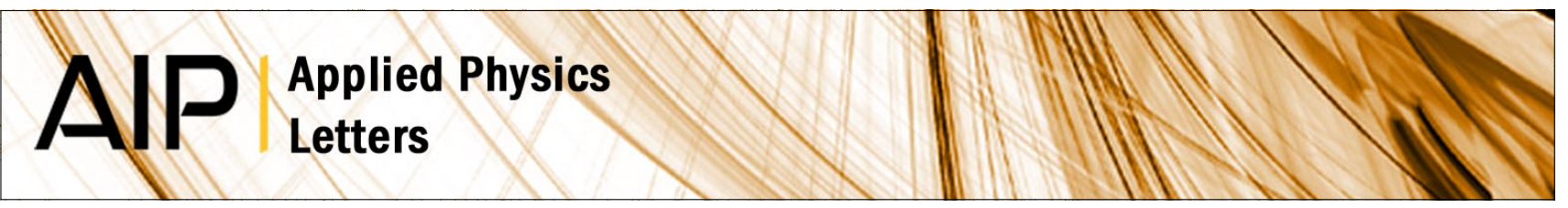

\title{
Superradiant lasing from J-aggregated molecules adsorbed onto colloidal silver
}

Serdar Özçelik, Isin Özçelik, and Daniel L. Akins

Citation: Appl. Phys. Lett. 73, 1949 (1998); doi: 10.1063/1.122563

View online: http://dx.doi.org/10.1063/1.122563

View Table of Contents: http://apl.aip.org/resource/1/APPLAB/v73/i14

Published by the American Institute of Physics.

\section{Related Articles}

Active subnanometer spectral control of a random laser Appl. Phys. Lett. 102, 071105 (2013)

Design, modeling, and performance evaluation of a novel dye cell for a high repetition rate dye laser Rev. Sci. Instrum. 83, 105114 (2012)

Emission wavelength of multilayer distributed feedback dye lasers

Appl. Phys. Lett. 101, 151123 (2012)

Optofluidic random laser

Appl. Phys. Lett. 101, 151101 (2012)

Long-range surface plasmon single-mode laser concepts

J. Appl. Phys. 112, 063115 (2012)

\section{Additional information on Appl. Phys. Lett.}

Journal Homepage: http://apl.aip.org/

Journal Information: http://apl.aip.org/about/about_the_journal

Top downloads: http://apl.aip.org/features/most_downloaded

Information for Authors: http://apl.aip.org/authors

\section{ADVERTISEMENT}

\section{AIP Applied Physics Letters}

\section{EXPLORE WHAT'S NEW IN APL}

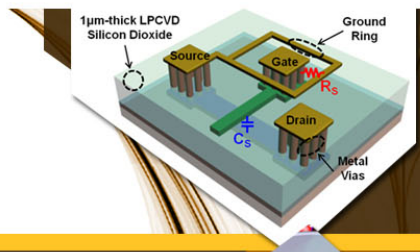

SURFACES AND INTERFACES

Focusing on physical, chemical, biological, structural, optical, magnetic and electrical properties of surfaces and interfaces, and more.. 


\title{
Superradiant lasing from $\mathrm{J}$-aggregated molecules adsorbed onto colloidal silver
}

\author{
Serdar Özçelik, ${ }^{\text {a) }}$ Isin Özçelik, ${ }^{\text {b) }}$ and Daniel L. Akins ${ }^{\text {c) }}$ \\ Center for Analysis of Structures and Interfaces (CASI), Department of Chemistry, \\ The City College of The City University of New York, New York, New York 10031
}

(Received 21 May 1998; accepted for publication 3 August 1998)

\begin{abstract}
The picosecond time-resolved emission spectrum of the cyanine dye 1,1'-diethyl-3,3'bis-(3-sulfopropyl)-5,5',6,6'-tetrachlorobenzimidazolocarbocyanine (also known as BIC) adsorbed onto colloidal silver was examined as a function of laser pulse energy at room temperature. BIC is found to aggregate on colloidal silver, and the number of coherently responding molecules involved in the one-exciton state (i.e., the coherence length) was estimated to involve 8-9 molecules. Lasing at a remarkably low incident pulse energy threshold was found for this system and explained in terms of a mechanism involving superradiant states created in coherently coupled adsorbed molecules that emit photons which stimulate emission from other spatially distributed superradiant states. (C) 1998 American Institute of Physics. [S0003-6951(98)03140-4]
\end{abstract}

The formulation of nanostructural devices from molecules, i.e., molecular nanoscale devices, promises the same potential photonic/optoelectronic applications as the more conventional systems (such as those formed using epitaxially prepared inorganic semiconductor superlattices or conjugated organic polymers), e.g., as nanoscale light emitters, as nonlinear light manipulators, as nanoscale sites in matrices for optical storage, as light emitting diodes, etc., as well as special opportunities for applications as prosthetic devices in molecular environments. ${ }^{1}$ Additionally, the use of molecules as building blocks for nanoscale devices is particularly attractive since their inherent quantum energy states can be expected to play a fundamental and flexible role in determining device properties. ${ }^{1}$

Many photoinduced properties of molecules can be exploited for device applications, including electron conduction by linked molecules, intramolecular/intermolecular electron transfer, conformational switching, and rapid changes in absorption/emission. Quite often, molecular excitons play a fundamental role in such processes.

Excitons can interact with each other and/or the surroundings resulting in complex dynamics. At low exciton density, one expects to observe single emission bands with monoexponential decay. At high exciton density, however, multiemission bands with nonmonoexponential decays are to be expected. Such behavior results from creation of higher multiexciton states and exciton-exciton interactions, both of which have been assumed to play a role in exciton-exciton annihilation. ${ }^{2-6}$ Recent time-resolved, wavelength-resolved emission studies from this laboratory have shown, in fact, that exciton annihilation can be quite complex, depending on both optical and population dynamics. ${ }^{7}$ Specifically, exciton dynamics has been discussed in terms of the existence of, so-called, one and two excitons that occupy states within the one- or two-exciton manifolds, respectively, ${ }^{7}$ in line with the

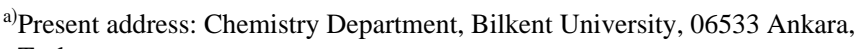
Turkey.

${ }^{b)}$ Present address: Tubitak-National Academic Computer and Information Center, Ankara, Turkey.

${ }^{c}$ Person to whom reprint requests should be sent. Electronic mail: akins@scisun.sci.ccny.cuny.edu
}

original description of the phenomenon by others. ${ }^{3,5,8}$ The one-exciton band of states result from the $\mathrm{N}$ molecules of the aggregate sharing one single-molecule excitation, while in the two-exciton band, $\mathrm{N}$ molecules of the aggregate share two single-molecule excitations. ${ }^{3,9}$ We have suggested that a key element of the optical and population dynamic schemes which explains exciton-exciton annihilation is the stimulation of one-exciton emission by photons derived from the transition between the two-exciton state and the one-exciton state. $^{7,10}$

Also recently, in a paper from this laboratory, we reported on an extremely low threshold for lasing for a system composed of the cyanine dye TTBC (specifically, 1,1'-3,3'-tetraethyl-5,5',6,6' 'tetrachlorobenzimidazolocarbocyanine iodide) adsorbed onto colloidal silica. ${ }^{10}$ Absorption spectra were interpreted as indicating that aggregate domains (coherently responding subgroups of molecules along the physical length of the aggregate) form on the colloidal, while fluorescence lifetime measurements indicate that the emission is superradiant. In the model developed to explain optical dynamics, the superradiant emission was conjectured to stimulate superradiance from other spatially distributed domains on the colloidal particles, and to result in an extremely low threshold for lasing, estimated to be a factor of $3 \times 10^{4}$ times smaller than any reported in the literature! ${ }^{10}$

We have advanced the idea that the superradiant lasing mechanism, which differs from more common four-level lasing schemes, ${ }^{11,12}$ as for example used to explain amplified spontaneous emission (ASE) ${ }_{13}^{13}$ applies for aggregated molecules dispersed throughout an excited medium.

In this letter, a report is provided on the exciton dynamics of a $J$-aggregated cyanine adsorbed onto colloidal silver particles. This study makes use of analyses of time-resolved, wavelength-resolved, and incident intensity dependent emission spectra. The specific focus of this work is the potassium salt of 1,1'-diethyl-3,3'-bis-(3-sulfopropyl)-5,5', 6,6'-tetrachloro-benzimidazolocarbocyanine (BIC; see Fig. $1)$.

All spectroscopic measurements were conducted at room temperature. BIC was purchased from Molecular Probe, Eugene, OR, and used without further purification. Aqueous 

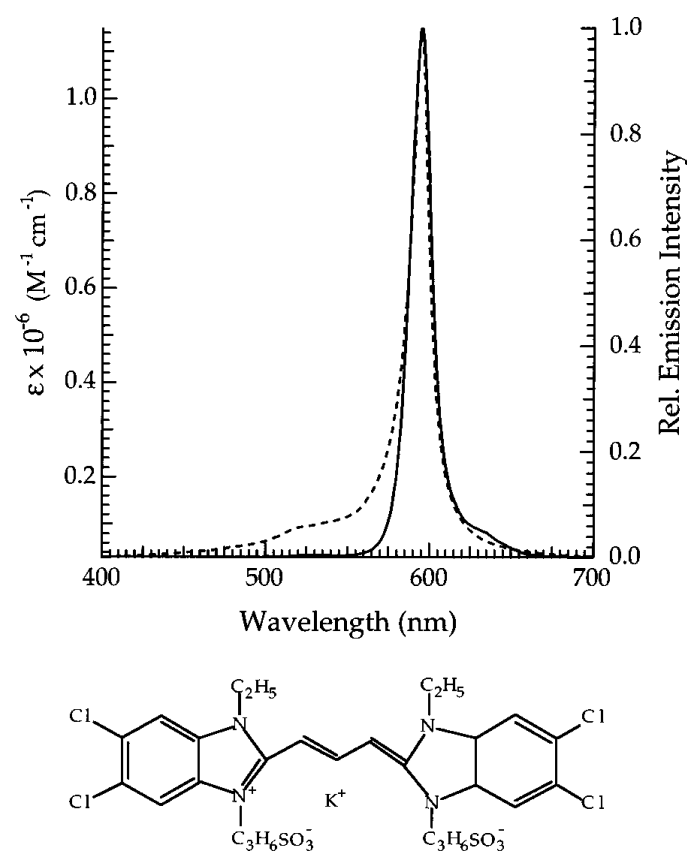

FIG. 1. Absorption and emission spectra of $J$-aggregated BIC adsorbed onto a silver colloid surface.

colloidal silver suspension was prepared by reduction of 2

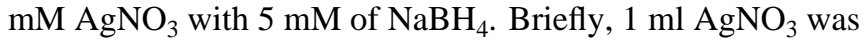
dropwise added into $50 \mathrm{ml}$ of $\mathrm{NaBH}_{4}$, with vigorous stirring. This mixture was stirred for $1 \mathrm{~h}$ in an ice bath. The resultant silver sol had an absorption peak of $\sim 382 \mathrm{~nm}$, was yellow in color, and stable for several days. Similar preparations have been shown by transmission electron microscopy (TEM) or scanning electron microscopy (SEM) to be essentially spherical with diameter ranging from 10 to $50 \mathrm{~nm} .{ }^{14,15}$

In order to prepare $J$ aggregates, $1 \mathrm{ml}$ of $1 \mathrm{mM} \mathrm{BIC}$ in methanol was mixed with $4 \mathrm{ml}$ of aqueous silver colloid. Such samples were excited using various incident intensities supplied by a picosecond Nd:YAG laser; see below. The intensity of the incident radiation was adjusted through the use of calibrated neutral density filters. Front-surface luminescence measurements, utilizing a $1 \mathrm{~mm}$ thickness fluorescence cell and an optical fiber for emission collection, were conducted.

Absorption spectra were recorded using a Perkin-Elmer Lambda 18, UV-vis/near-IR spectrometer. Steady-state fluorescence spectra were acquired using a SPEX Fluorolog- $\tau 2$ spectrofluorometer. Time-dependent emission measurements utilized a Hamamatsu streak camera, Model C4334, optically coupled to a charge-coupled-device (CCD) array detector. This system allowed the measurements of both the emission decay rate and the time-resolved emission spectrum. For this latter study a Chromex 205i imaging spectrometer was used. The ultimate time resolution which we have been able to attain with this system, using Hamamatsu U4290 fluorescence analysis software, is estimated to be $\sim 10$ ps.

The exciting light source for the emission studies was a Coherent 702 mode locked, picosecond dye laser of $10 \mathrm{ps}$ pulse width and $76 \mathrm{MHz}$ repetition rate, pumped by a Coherent Antares 76s Nd:YAG laser. The excitation wavelength, which overlaps the $J$-aggregate absorption (see Fig. 1), was $\sim 570 \mathrm{~nm}$. For the present study, a high-pass filter (transmitting for wavelengths greater than $580 \mathrm{~nm}$ ) was placed at the entrance slit of the Chromex spectrometer.

Figure 1 shows the absorption and emission spectra of $J$-aggregated BIC. Both spectra have band maxima at $\sim 595$ nm.

For the emission, reabsorption effects have been minimized by using a $1 \mathrm{~mm}$ optical path length cell and frontface detection. The excitation spectrum (not shown) was found to be essentially identical to the absorption spectrum, confirming that reabsorption effects were effectively absent.

Figure 2 shows emission decays as a function of incident excitation intensity. For all measurements, unfocused laser radiation was used. At the lowest incident intensity, $0.2 \mathrm{pJ} /$ pulse, a single exponential decay is observed; with decay time of $149.5 \pm 1.4$ ps. We have assigned this value as the decay time of the one-exciton state. A similar observation has been made by Yoshihara and co-workers ${ }^{16-18}$ for the sodium salt of BIC. The difference in alkali counter ion used in this work and that of Yoshihara and co-workers $\left(\mathrm{K}^{+}\right.$and $\mathrm{Na}^{+}$, respectively) as well as difference in the nature of the systems (dye adsorbed onto colloidal silver particles, and aggregate formed in a low-temperature water/glycol solution, respectively) are sufficient to rationalize difference in oneexciton decay rate for BIC in the two environments.

From the one-exciton decay time that we measured, one can estimate an upper limit for the coherent length of the aggregate (at room temperature) assuming the decay time corresponds to the radiative lifetime, and using the reported radiative rate constant $\left(7.8 \times 10^{8} \mathrm{~s}^{-1}\right)^{17}$ of the monomeric sodium salt of BIC. The ratio of radiative rate constants suggests that the coherence length involves 8-9 molecules.

The emission decay profile changes when incident excitation intensity is increased (see Fig. 2). At $\sim 2$ pJ/pulse, an additional narrower emission feature, which has a faster decay rate than the one-exciton state, appears early in the emission relaxation. An emission decay component that has the same decay time as indicated above for the one-exciton state also exists. We interpret the additional emission feature as revealing the existence of a coherent emission from the $J$ aggregate/colloid system which is distinct from the superradiance associated with the coherently responding molecules that define the excitonic state. The pulse width of this putative coherent emission is estimated to be $\sim 40 \mathrm{ps}$ for the $2 \mathrm{pJ}$ incident pulse. It is to be noted that the presence of coherent emission is likely not the result of a population inversion involving the one-exciton state since resonance trapping, attributable to the resonance overlap of the one-exciton emission and absorption (see Fig. 1), would make it impossible for there to be a net stimulated emission gain path length through the sample required for amplification of one-exciton photons. The front-face detection geometry further indicates the unimportance of gain path length involving one-exciton population in promoting stimulated emission.

When the incident intensity was set to $\sim 500 \mathrm{pJ} /$ pulse (see Fig. 2 where excitation of $526 \mathrm{pJ} /$ pulse was used), a single, temporally sharp emission was found, with a pulse width of $\sim 12 \mathrm{ps}$. This sharp temporal profile is attributed by us (vide infra) to a combination of gain narrowing and the intrinsic narrowness of the emission associated with the transition between the two- and one-exciton states. ${ }^{7}$

Figure 3 shows time-resolved emission spectra at differ- 


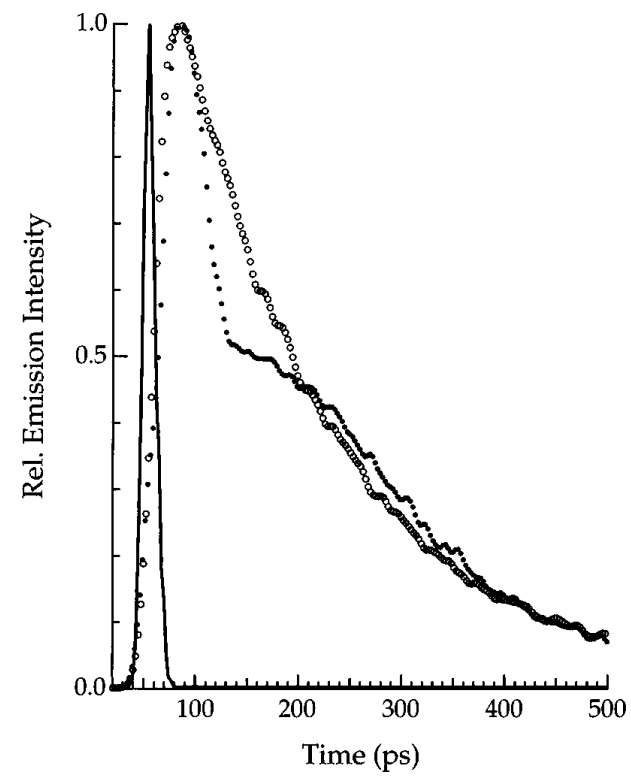

FIG. 2. Emission decay of aggregated BIC adsorbed onto a silver colloid surface for incident pulse intensities: $0.2 \mathrm{pJ} / \mathrm{pulse}$ (open circle); $2.0 \mathrm{pJ} / \mathrm{pulse}$ (dot); $525 \mathrm{pJ} /$ pulse (solid line).

ent incident intensities for BIC. At the lowest excitation energy $(0.2 \mathrm{pJ} /$ pulse $)$ the spectrum is determined to be composed of emissions from two components, attributed to monomers and aggregates.

It is to be noted that the coherent emission has been observed only when aggregates form on the colloid surface, ${ }^{7}$ and was not observed at incident excitation intensity up to $1.0 \mathrm{~nJ} /$ pulse for $J$ aggregates formed in aqueous homogeneous solutions. ${ }^{19}$

The mechanism for laserlike emission by BIC on colloidal silver is presumed to consist of the same essential features as suggested by us for aggregated TTBC adsorbed onto silica. To wit, photons resulting from a transition between the two-exciton state and the one-exciton state (viz., two-toone photons) stimulate one-exciton photon transitions, thus amplifying the photon flux at the expense of one-exciton population density, and leading to lasing at a low excitation threshold. The fact that the two-to-one emission is essentially at the same wavelength (see Fig. 3) as that of the one-to-zero exciton emission (see Fig. 1) points out that it is the temporal distinction between the two emissions that leads to the photons derived from the two-to-one process determining the optical dynamics. The wavelengths of the two emissions are important only in that there must be an overlap of emissions, leading to the population of the "prey" state (i.e., the oneexciton state) being controlled by the "predator" state's (i.e., the two-exciton state) photons. This latter assessment is a refining of the earlier suggestion that the wavelengths had to differ. ${ }^{10}$

We thus conclude that key ingredients that lead to laserlike emission for the BIC/colloidal silver system include the superradiant emission and the collective, stimulated emission of distributed excited aggregate domains throughout the total excited volume.

It is to be noted that the threshold for lasing for this system corresponds to $\sim 2 \mathrm{pJ} /$ pulse, a phenomenally low value; in fact, a factor of $\sim 20$ less than that reported earlier for TTBC. ${ }^{10}$ This system, therefore has a threshold that is

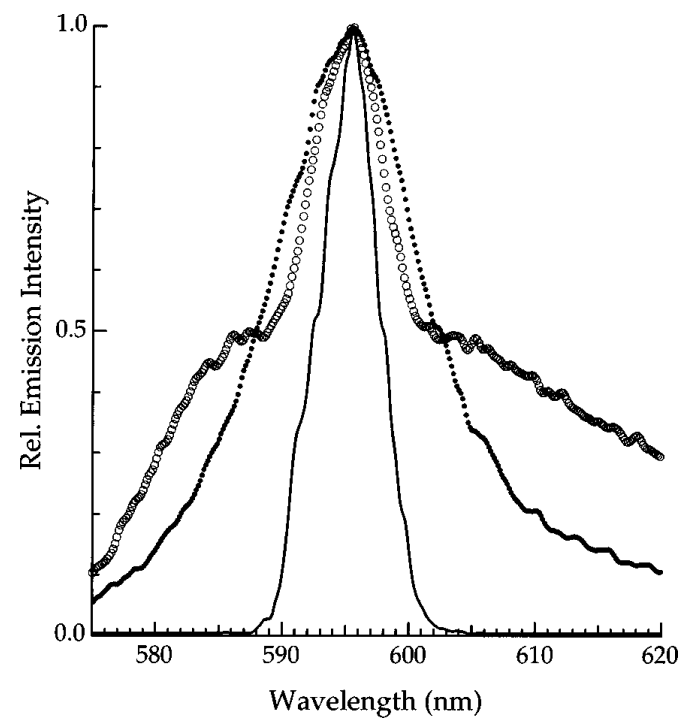

FIG. 3. Time-resolved, wavelength-resolved emission spectrum of adsorbed, aggregated BIC, with a 25 ps time window. Incident pulse intensities are the same as in Fig. 2.

smaller than the lowest lasing threshold reported in the literature by a factor of $6 \times 10^{5}$ !

Given the $J$-aggregate character of the emission and absorption, the adsorbed dye molecules would appear to align themselves on the adsorbate surface in such a fashion so as to form whispering gallery mode microcavities. ${ }^{20}$ In fact, the nanometer and possibly subnanometer emission active layer would suggest describing it as a "nanocavity."

Support for this research by the National Science Foundation (NSF) under Grant No. HRD-9353488 and by NASA under its FAR Program (Grant NAG5-4019) are gratefully acknowledged.

${ }^{1}$ R. C. Haddon and A. A. Lamola, Proc. Natl. Acad. Sci. USA 82, 1874 (1985).

${ }^{2}$ V. Sundström, T. Gillbro, R. A. Gadonas, and A. Piskarskas, J. Chem. Phys. 89, 2754 (1988).

${ }^{3}$ M. van Burgel, D. A. Wiersma, and K. Duppen, J. Chem. Phys. 102, 20 (1995).

${ }^{4}$ D. V. Braumbaugh, A. A. Muenter, W. Knox, G. Mourau, and B. Wittmershaus, J. Lumin. 31, 783 (1984).

${ }^{5}$ A. E. Johnson, S. Kumazaki, and K. Yoshihara, Chem. Phys. Lett. 211, 511 (1993).

${ }^{6}$ P. J. Reid, D. A. Higgins, and P. F. Barbara, J. Phys. Chem. 100, 3892 (1996).

${ }^{7}$ D. L. Akins and S. Özçelik, J. Phys. Chem. B 101, 3021 (1997).

${ }^{8}$ H. Fidder, J. Knoester, and D. A. Barbara, J. Chem. Phys. 98, 6564 (1993).

${ }^{9}$ J. Knoester and F. C. Spano, in J-Aggregates, edited by T. Kobayashi (World Scientific, Singapore, 1996), pp. 111-160.

${ }^{10}$ S. Özçelik and D. L. Akins, Appl. Phys. Lett. 71, 3057 (1997).

${ }^{11}$ A. U. Khan and M. Kasha, Proc. Natl. Acad. Sci. USA 80, 1767 (1983).

${ }^{12}$ P. Chou, D. McMorrow, T. J. Asrtsma, and M. Kasha, J. Phys. Chem. 88, 4596 (1984).

${ }^{13}$ C. V. Shank, Rev. Mod. Phys. 47, 649 (1975).

${ }^{14}$ A. J. Henglein, J. Chem. Phys. 83, 2209 (1979).

${ }^{15}$ A. M. Ahern and R. L. Garrell, Anal. Chem. 59, 2816 (1987).

${ }^{16}$ V. F. Kamalov, I. A. Struganova, Y. Koyama, and K. Yoshihara, Chem. Phys. Lett. 220, 257 (1994).

${ }^{17}$ V. F. Kamalov, I. A. Struganova, Y. Koyama, and K. Yoshihara, Chem. Phys. Lett. 226, 132 (1994)

${ }^{18}$ V. F. Kamalov, I. A. Struganova, and K. Yoshihara, J. Phys. Chem. 100, 8640 (1996).

${ }^{19} \mathrm{~S}$. Özçelik and D. L. Akins (unpublished).

${ }^{20}$ Y. Yamamoto and R. E. Slusher, Phys. Today 46, 66 (1993). 